\title{
THE APPROXIMATE SOLUTION OF CERTAIN NONLINEAR DIFFERENTIAL EQUATIONS ${ }^{1}$
}

\author{
R. G. HUFFSTUTLER AND F. MAX STEIN
}

1. Introduction. We consider the best approximation by polynomials $P_{n}(x)$ of the solution on $[0,1]$, denoted by $I$, of the first order nonlinear differential equation,

$$
L(y) \equiv y^{\prime}-\sum_{k=1}^{m} f_{k}(x) y^{k}=R(x),
$$

in a manner somewhat analogous to that used by McEwen [4], Oberg [5], and Stein [6], in the sense that

$$
\left\|R(x)-L\left[P_{n}(x)\right]\right\|=\sup _{0 \leqq x \leqq 1}\left|R(x)-L\left[P_{n}(x)\right]\right|
$$

is a minimum. The operators $L(y)$ used in [4], [5], [6] were all linear and the minimizing condition $\int_{a}^{b}\left|R(x)-L\left(P_{n}\right)\right|^{a} d x$ was used in place of (2); since $L(y)$ in (1) is nonlinear, different analysis must be applied.

We assume that the coefficients in (1) are such that there exists a unique solution $y(x)$ which satisfies the boundary condition

$$
y(0)=0 .
$$

2. Existence of minimizing polynomials. The existence of polynomials that make (2) a minimum and which satisfy (3) can be proved in a manner analogous to that used by Jackson [2]; the theorem giving this result is stated without proof in terms of a slightly more general boundary condition and in terms of general linearly independent functions rather than polynomials.

THEOREM 1. Let (1) be subject to the boundary condition $y_{0}=y\left(x_{0}\right)$, $x_{0} \in[a, b]$, with $R(x)$ and $f_{k}(x), k=1,2, \cdots, m$, continuous on $[a, b]$, and suppose that $\left\{\Phi_{k}(x)\right\}$ is a set of $n$ differentiable functions whose set of first derivatives $\left\{\Phi_{k}^{\prime}\right\}$ is a set of continuous functions on $[a, b]$. Suppose that $f_{m}(x)$ and the set of functions $\left\{\Phi_{k}(x)\right\}$ satisfy the conditions that $f_{m}(x) \neq 0$ almost everywhere on $[a, b]$ and the set $\left\{\Phi_{k}(x)\right\}$ are linearly independent on $[a, b]$. Then there exists a linear combination $S_{n}(x)$ of the elements of $\left\{\Phi_{k}(x)\right\}$ which satisfies the boundary condition and, at the same time, minimizes (2) for all such sums of this type.

Presented to the Society, April 22, 1967; received by the editors April 18, 1967.

1 This work was partially supported by AFOSR Grants 185-65 and 67. 
In the case of polynomials the interval is $[0,1]$ and the set of linearly independent functions is $\left\{x^{k}\right\}, k$ a nonnegative integer. With the imposed boundary condition (3), we are restricted to considering polynomials of the type

$$
P_{n}(x)=\sum_{k=1}^{n} a_{k} x^{k}
$$

The need for this particular boundary condition will be evident in the work that follows.

3. The approximate solution of the differential equation. In order to arrive at the principal result of the paper, we need a theorem given by Coppel [2], which we likewise state without proof.

TheOREM 2. Let $\left\{f_{n}(x, y)\right\}$ be a sequence of functions which are defined and continuous in an open set $D$, and suppose that

$$
\lim _{n \rightarrow \infty} f_{n}(x, y)=f(x, y)
$$

exists uniformly on any compact subset of $D$. Let $\left(x_{n}, y_{n}\right)$ be a sequence of points converging to a point $\left(x_{0}, y_{0}\right)$ of $D$, and let $\Phi_{n}(x)$ be the unique solution of the differential equation $y^{\prime}=f_{n}(x, y)$ which passes through the point $\left(x_{n}, y_{n}\right)$. If the solution $\Phi(x)$ of the differential equation $y^{\prime}=f(x, y)$ which passes through the point $\left(x_{0}, y_{0}\right)$ is defined on the interval $[a, b]$ and is unique, then $\Phi_{n}(x)$ is defined on $[a, b]$ for all sufficiently large $n$, and $\Phi_{n}(x) \rightarrow \Phi(x)$ uniformly on this interval as $n \rightarrow \infty$.

We now consider the approximate solution of (1) by polynomials of the form (4) which minimize (2). That is, we determine the conditions under which the polynomials of the form (4) which minimize (2) will converge uniformly to the solution $y(x)$ of (1) on $I$.

Let $y(x)$ be the unique solution of (1) which satisfies the boundary condition (3). Then, by an extension of the Weierstrass theorem, we know that for any positive $\eta<1$ there exists at least one polynomial $s_{n}(x)$ of the form (4) of some degree $n$ such that

$$
\left|y^{(k)}(x)-s_{n}^{(k)}(x)\right| \leqq \eta, \quad k=0,1 .
$$

Corresponding to the degree $n$ of $s_{n}(x)$, there is a polynomial $P_{n}(x)$ of degree $n$ at most which is of the form (4) and which makes (2) a minimum.

We now let $r(x)=y(x)-s_{n}(x)$ and $Q_{n}(x)=P_{n}(x)-s_{n}(x)$, where $y(x)$, $s_{n}(x)$, and $P_{n}(x)$ have been defined above. Thus we have 


$$
\begin{aligned}
\gamma & =\left\|R(x)-L\left[P_{n}(x)\right]\right\|=\left\|L(y)-L\left(P_{n}\right)\right\| \\
& =\left\|L\left(y-P_{n}\right)+\sum_{k=1}^{m} f_{k}\left(y-P_{n}\right)^{k}-\sum_{k=1}^{m} f_{k}\left(y^{k}-P_{n}^{k}\right)\right\| .
\end{aligned}
$$

But since $y^{k}-P_{n}^{k}=y^{k-1}\left(y-P_{n}\right)+P_{n}\left(y^{k-1}-P_{n}^{k-1}\right)$, then (7) becomes

$$
\begin{aligned}
\gamma=\| L\left(y-P_{n}\right) & +\sum_{k=1}^{m} f_{k}\left(y-P_{n}\right)^{k} \\
& \quad-\sum_{k=1}^{m} f_{k}\left[y^{k-1}+y^{k-2} P_{n}+\cdots+P_{n}^{k-1}\right]\left(y-P_{n}\right) \| \\
=\| L\left(r-Q_{n}\right) & +\sum_{k=1}^{m} f_{k}\left(r-Q_{n}\right)^{k} \\
& \quad-\sum_{k=1}^{m} f_{k}\left[y^{k-1}+y^{k-2} P_{n}+\cdots+P_{n}^{k-1}\right]\left(r-Q_{n}\right) \| \\
\leqq & \left\|L(r)+\sum_{k=1}^{m} f_{k} r^{k}-\sum_{k=1}^{m} f_{k}\left[y^{k-1}+y^{k-2} s_{n}+\cdots+s_{n}^{k-1}\right] r\right\|,
\end{aligned}
$$

where the last step is permissible since $P_{n}(x)$ is the minimizing polynomial for $y(x)$, and the value of the norm is no less for any other sum.

For $x \in I$, let $C=\max \left\{1,|y|,|y|^{2}, \cdots,|y|^{m-1}\right\}$ and $M_{1}$ $=\max \left\{\left|f_{k}(x)\right|\right\}, k=1,2, \cdots, m$, and let $A=\max \left\{1,\left|s_{n}(x)\right|\right\}$. Then

$$
\begin{aligned}
\gamma & \leqq L(r)\left\|+\sum_{k=1}^{m}\right\| f_{k} r^{k}\left\|+\sum_{k=1}^{m}\right\| f_{k}\left[y^{k-1}+y^{k-2} s_{n}+\cdots+s_{n}^{k-1}\right] r \| \\
& \leqq\|L(r)\|+\sum_{k=1}^{m} M_{1} \eta^{k}+\sum_{k=1}^{m} M_{1}\left[C+C A+\cdots+C A^{k-1}\right] \eta \\
& \leqq\|L(r)\|+m M_{1} \eta+m(m-1) M_{1} A^{m-1} C \eta,
\end{aligned}
$$

where use has been made of the facts that $\eta<1, A>1$, and $|r|$ $=\left|y-s_{n}\right| \leqq \eta$. Now

$$
|L(r)| \leqq\left|r^{\prime}\right|+\sum_{k=1}^{m}\left|f_{k} \| r\right|^{k} \leqq \eta+\sum_{k=1}^{m} M_{1} \eta^{k} \leqq M_{2} \eta,
$$

by again making use of the fact that $\eta<1$. Thus

$$
\|L(r)\|=\sup _{0 \leqq x \leqq 1}|L(r)| \leqq M_{2} \eta .
$$


Finally,

$$
\gamma \leqq M_{2} \eta+M_{1} m \eta+M_{1} C m(m-1) A^{m-1} \eta \leqq M_{3} \eta,
$$

where $M_{3}$ is a positive constant. That is, given a null sequence $\left\{\eta_{k}\right\}, k=1,2, \cdots$, with $\eta_{1}<1, L\left(P_{n}\right)$ converges uniformly to $L(y)$.

Now let $y(x)-P_{n}(x)=w_{n}(x)$ and let $L(y)-L\left(P_{n}\right)=F_{n}(x)$ for $x \in[0,1]$. Then

$$
\begin{aligned}
F_{n}(x) & =L(y)-L\left(P_{n}\right)=L(y)-L\left(y-w_{n}\right) \\
& =y^{\prime}-\sum_{k=1}^{m} f_{k} y^{k}-\left(y^{\prime}-w_{n}^{\prime}\right)+\sum_{k=1}^{m} f_{k}\left(y-w_{n}\right)^{k} \\
& =w_{n}^{\prime}-\sum_{k=1}^{m} h_{k} w_{n}^{k},
\end{aligned}
$$

where the $h_{k}$ 's are products of the known functions $y$ and $f_{k}$ and their various powers up to the power $m$. Therefore we have reduced the problem to one of considering differential systems of the form

$$
w^{\prime}=\sum_{k=1}^{m} h_{k} w^{k}+F_{n}(x), \quad w(0)=0 .
$$

Note that $F_{n}(x)$ converges uniformly to zero throughout $[0,1]$, since $\left\|F_{n}(x)\right\|=\left\|L(y)-L\left(P_{n}\right)\right\| \leqq M_{3} \eta$, and $\eta$ can be chosen arbitrarily small.

From (10) we have that

$$
\lim _{n \rightarrow \infty}\left[\sum_{k=1}^{m} h_{k} w^{k}+F_{n}(x)\right]=\sum_{k=1}^{m} h_{k} 2 w^{k}
$$

uniformly throughout $[0,1]$, since $F_{n}(x)$ converges uniformly to zero in this interval. The unique solution of

$$
w^{\prime}=\sum_{k=1}^{m} h_{k} w^{k}, \quad w(0)=0
$$

is $w(x) \equiv 0, x \in[0,1]$. Hence, by Theorem 2, (although Theorem 2 is stated in terms of an open set, it remains valid for our restricted application), the solutions of (10) converge uniformly to the zero solution throughout $[0,1]$. Thus,

$$
\lim _{n \rightarrow \infty}\left|y(x)-P_{n}(x)\right|=\lim _{n \rightarrow \infty}\left|w_{n}(x)\right|=0, \quad x \in[0,1],
$$

where $w_{n}(x)$ is the unique solution of $(10)$ for the corresponding value of $n$. That is, the minimizing polynomials $\left\{P_{n}(x)\right\}$ converge uni- 
formly throughout $[0,1]$ to the solution $y(x)$ of (1).

If we consider again $L(y)-L\left(P_{n}\right)=F_{n}(x)$, we have that

$$
y^{\prime}-P_{n}^{\prime}-\sum_{k=1}^{m} f_{k}\left(y^{k}-P_{n}^{k}\right)=F_{n}(x),
$$

or

$$
y^{\prime}-P_{n}^{\prime}=\left(y-P_{n}\right) G\left(P_{n}, y\right)+F_{n}(x),
$$

where $G\left(P_{n}, y\right)$, containing the $f_{k}$ 's, is a continuous function of $P_{n}(x)$ and $y(x)$ on $[0,1]$. It follows from the uniform convergence of the set $\left\{P_{n}(x)\right\}$ to $y(x)$ that the $P_{n}$ 's are uniformly bounded on $[0,1]$. Therefore, since $y(x)$ is bounded on $[0,1]$ and $P_{n}(x)$ is bounded on $[0,1],\left|G\left(P_{n}, y\right)\right| \leqq M_{4}$ for all $x \in[0,1]$.

Given an $\epsilon>0$, there exists an $N_{1}$ such that for $n \geqq N_{1},\left|F_{n}(x)\right|$ $\leqq \epsilon / 2$, since $F_{n}(x)$ converges uniformly to zero. Likewise, there exists an $N_{2}$ such that for $n \geqq N_{2},\left|y-P_{n}\right| \leqq \epsilon / 2 M_{4}$. Then for $n \geqq \max \left\{N_{1}, N_{2}\right\}$ we have that

$$
\begin{aligned}
\left|y^{\prime}-P_{n}^{\prime}\right| & \leqq\left|y-P_{n} \| G\left(P_{n}, y\right)\right|+\left|F_{n}(x)\right| \\
& \leqq\left(\epsilon / 2 M_{4}\right) M_{4}+\epsilon / 2=\epsilon, \quad x \in I .
\end{aligned}
$$

Hence, the sequence $\left\{P_{n}^{\prime}\right\}$ converges uniformly throughout $[0,1]$ to $y^{\prime}(x)$, the derivative of the solution of (1).

The preceding discussion may be summarized in the following theorem.

THEOREM 3. If $y(x)$ is the unique solution of (1) which satisfies the boundary condition (3), and if $P_{n}(x)$ is a polynomial of the form (4) which minimizes (2), then $P_{n}(x)$ and $P_{n}^{\prime}(x)$ converge uniformly throughout $[0,1]$ to $y(x)$ and $y^{\prime}(x)$ respectively as $n$ increases without bound.

\section{REFERENCES}

1. E. A. Coddington and N. Levinson, Theory of ordinary differential equations, McGraw-Hill, New York, 1955.

2. W. A. Coppel, Stability and asymptotic behavior of differential equations, Heath, Boston, 1965.

3. D. Jackson, On functions of closest approximation, Trans. Amer. Math. Soc. 22 (1921), 117-128.

4. W. H. McEwen, Problems of closest approximation connected with the solution of linear differential equations, Trans. Amer. Math. Soc. 33 (1931), 979-997.

5. E. N. Oberg, The approximate solution of integral equations, Bull. Amer. Math. Soc. 41 (1935), 276-284.

6. F. Max Stein, The approximate solution of integro-differential equations, Ph.D. dissertation, State Univ. of Iowa, Iowa City, 1955, pp. 1-66. 